\title{
Observation of Electromagnetically Induced Transparency
}

\author{
K.-J. Boller, A. Imamoğlu, and S. E. Harris \\ Edward L. Ginzton Laboratory, Stanford University, Stanford, California 94305
}

(Received 12 December 1990)

\begin{abstract}
We report the first demonstration of a technique by which an optically thick medium may be rendered transparent. The transparency results from a destructive interference of two dressed states which are created by applying a temporally smooth coupling laser between a bound state of an atom and the upper state of the transition which is to be made transparent. The transmittance of an autoionizing (ultraviolet) transition in $\mathrm{Sr}$ is changed from $\exp (-20)$ without a coupling laser present to $\exp (-1)$ in the presence of a coupling laser.
\end{abstract}

PACS numbers: $42.50 . \mathrm{Qg}, 32.80 . \mathrm{Dz}, 42.50 . \mathrm{Hz}, 42.65 . \mathrm{Ky}$

This Letter reports the results of an experiment showing how an opaque atomic transition may be rendered transparent to radiation at its resonance frequency. This is accomplished by applying an electromagnetic coupling field between the upper state $|3\rangle$ of the transition and another state $|2\rangle$ of the atom (Fig. 1). When the Rabi frequency of the coupling field exceeds the inhomogeneous width of the $|1\rangle-|2\rangle$ transition, the medium becomes transparent on line center. In this work, the transmittance of an ultraviolet transition to an autoionizing state of neutral $\mathrm{Sr}$ is changed from $\exp (-20)$ without a coupling laser present to $\exp (-1)$ in the presence of a coupling laser.

The transparency described here is not a saturation or hole-burning phenomena. As the probe laser is turned on in the presence of the coupling laser, ground-state atoms evolve to a steady state where a fraction (in these experiments, about one part in a thousand) of their population is in state $|2\rangle$. This coherently phased population produces a dipole moment of equal magnitude and opposite sign to the primary dipole moment at the probe frequency. Equivalently, the transparency may be viewed as resulting from a combination of the ac-Stark splitting and the interference between the two dressed states which are created by the coupling laser [Fig. 1 (inset)]. It is the observation of the large change in opacity which results from this interference that is the special feature of this work.

There has been substantial theoretical work on laserinduced and laser-inhibited autoionization and the transparency observed here is a special case of the formalism and examples given by Lambropoulos, ${ }^{1}$ Eberly, ${ }^{2}$ Agarwal, ${ }^{3}$ and their co-workers. The related subject of laserinduced continuum structure has been reviewed by Knight, Lauder, and Dalton. ${ }^{4}$ Experimentally, ${ }^{5}$ resonances induced into a continuum by a laser have been shown to cause a polarization rotation, to increase the efficiency of third-harmonic generation, and to modify three-photon ionization. An interference in autoionization as caused by a dc electric field has been described. This interference is also of the same nature as that observed by Whitley and Stroud ${ }^{6}$ and Alzetta et al. ${ }^{6}$ in the
Rayleigh scattering of radiatively broadened systems.

Figure 1 shows a partial energy-level diagram of neutral strontium. ${ }^{7}$ The probe transition which is to be rendered transparent is $5 s 5 p^{1} P_{1}-4 d 5 d^{1} D_{2}$ with a transition wavelength of $337.1 \mathrm{~nm}$. The absorption oscillator strength of this transition, as calculated from the $R C N$ $R C G$ atomic physics code, ${ }^{8}$ is $g f=0.15$. The upper level of this transition decays by autoionization and has a measured width of $1.2 \mathrm{~cm}^{-1}$, corresponding to an autoionizing time of 4.4 psec. Its lower level is populated from ground by using a resonant $460.7-\mathrm{nm}$ pulsed dye
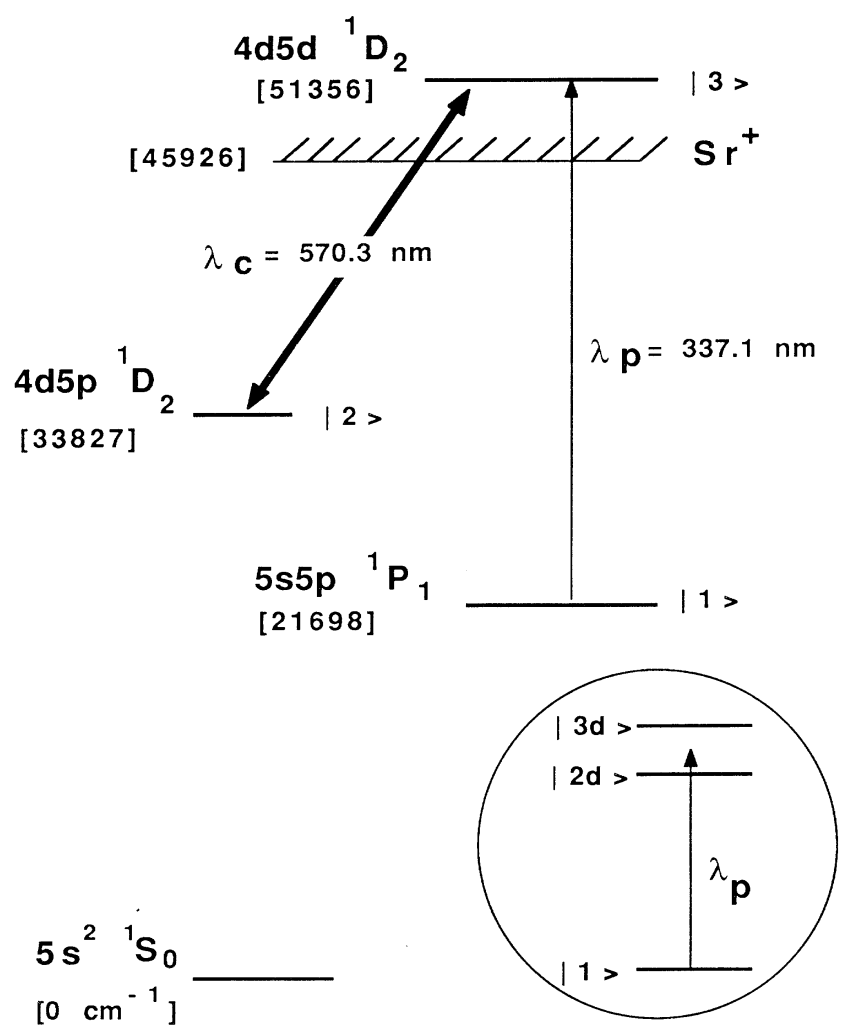

FIG. 1. Energy-level diagram of neutral Sr. Inset: Dressed-state picture. 
(pumping) laser. We use a ground-state $\left(5 s^{2}{ }^{1} S_{0}\right) \mathrm{Sr}$ density of $5 \times 10^{15}$ atoms $/ \mathrm{cm}^{3}$ and a pumping-laser energy density of about $10 \mathrm{~mJ} / \mathrm{cm}^{2}$ in a pulse width of 4 nsec, and obtain a population density of $5 s 5 p^{1} P_{1}$ of $1 \times 10^{14}$ atoms $/ \mathrm{cm}^{3}$. This population is radiatively trapped for about $50 \mathrm{nsec}$.

The laser which is used to create the transparency is termed as the coupling laser and is tuned to the line center $(570.3 \mathrm{~nm})$ of the $4 d 5 p^{1} D_{2}-4 d 5 d^{\prime} D_{2}$ transition $(g f=0.10)$. Both the coupling and probe laser beams are $\sigma^{+}$polarized, are copropagating, and in these experiments have intensities of $1.5 \times 10^{7}$ and $10^{4} \mathrm{~W} / \mathrm{cm}^{2}$, respectively.

The coupling-laser radiation is generated by a $(4.5 \mathrm{~cm}$ long) single-mode Littman dye laser ${ }^{9}$ which is pumped by a frequency-doubled injection-seeded (Quanta Ray) Nd-doped yttrium-aluminum-garnet (Nd:YAlG) laser. Although each pulse is near transform limited, changes in the refractive index of the dye cause a pulse-to-pulse frequency jitter corresponding to a time-averaged linewidth of $0.03 \mathrm{~cm}^{-1}$. Since the laser cavity mode does not track synchronously with the grating, the coupling laser, when run at maximum energy, may only be set within $0.2 \mathrm{~cm}^{-1}$. The Littman oscillator output is amplified by a three-stage dye amplifier to an energy of $20 \mathrm{~mJ}$ in a pulse length of $3 \mathrm{nsec}$ with a pulse-to-pulse peak intensity fluctuation of $10 \%$.

The probe laser is a frequency-doubled pressure-tuned Quanta Ray dye laser with an intracavity etalon, and is pumped by the same injection-seeded Quanta Ray laser as is the coupling laser. The energy, pulse length, and linewidth at $337.1 \mathrm{~nm}$ are $0.1 \mathrm{~mJ}, 3.5 \mathrm{nsec}$, and 0.15 $\mathrm{cm}^{-1}$. To avoid saturation, the output of this laser is attenuated by a factor of $10^{3}$.

The pumping dye laser at $460.7 \mathrm{~nm}$ was driven by the third harmonic of a different (nonseeded) Quanta Ray $\mathrm{Nd}$ :YAlG laser and had an energy and pulse length of about $1 \mathrm{~mJ}$ and $4 \mathrm{nsec}$.

All of the lasers were sent into a $\mathrm{Sr}$ heat pipe with a 10 -cm-long vapor zone. To ensure good spatial overlap of the coupling- and probe-laser beams, their beam diameters inside the $\mathrm{Sr}$ cell were 3 and $0.2 \mathrm{~mm}$, respectively. The diameter of the pumping-laser beam was $3 \mathrm{~mm}$. To ensure good temporal overlap, the transmitted probe beam is sampled by a fast photodiode (rise and fall time of $100 \mathrm{psec}$ ), and a fast gated integrator (gate width of 500 psec; Stanford Research Systems SR255). The delay of this gate is set so that the observed portion of the probe laser coincides with the temporally flat, maximum-intensity region of the coupling laser. Before beginning a transmission measurement, both the coupling and probe lasers are delayed by between 20 and $150 \mathrm{nsec}$ relative to the pumping laser. This delay is used, together with the pumping intensity, to set the population of the $5 s 5 p{ }^{1} P_{1}$ level.

Because the probe laser is multimode, its pulse-to- pulse intensity fluctuation during the 500-psec gated interval is nearly $100 \%$. Data are recorded by measuring the energy of the probe laser in the gated interval following the $\mathrm{Sr}$ cell, and the total energy (using a 20-nsec gate) before the $\mathrm{Sr}$ cell. A data point is obtained by averaging each for thirty pulses and dividing by an analog signal processor. To scan the probe laser over an 8$\mathrm{cm}^{-1}$ interval, with the lasers operating at $10 \mathrm{~Hz}$, typically requires $10 \mathrm{~min}$.

Figure 2 shows transmission as a function of the probe frequency, with the population of the $5 s 5 p^{1} P_{1}$ level small enough that the medium is still somewhat transparent on line center (delay of $110 \mathrm{nsec}$ ). Figure 2(a) shows the transmission profile with the coupling laser off. Here, the minimum transmission is $\exp (-1.7 \pm 0.1)$. By curve fitting (theory, dashed curve) with a Voigt profile convolved with the probe laser linewidth $(0.15$

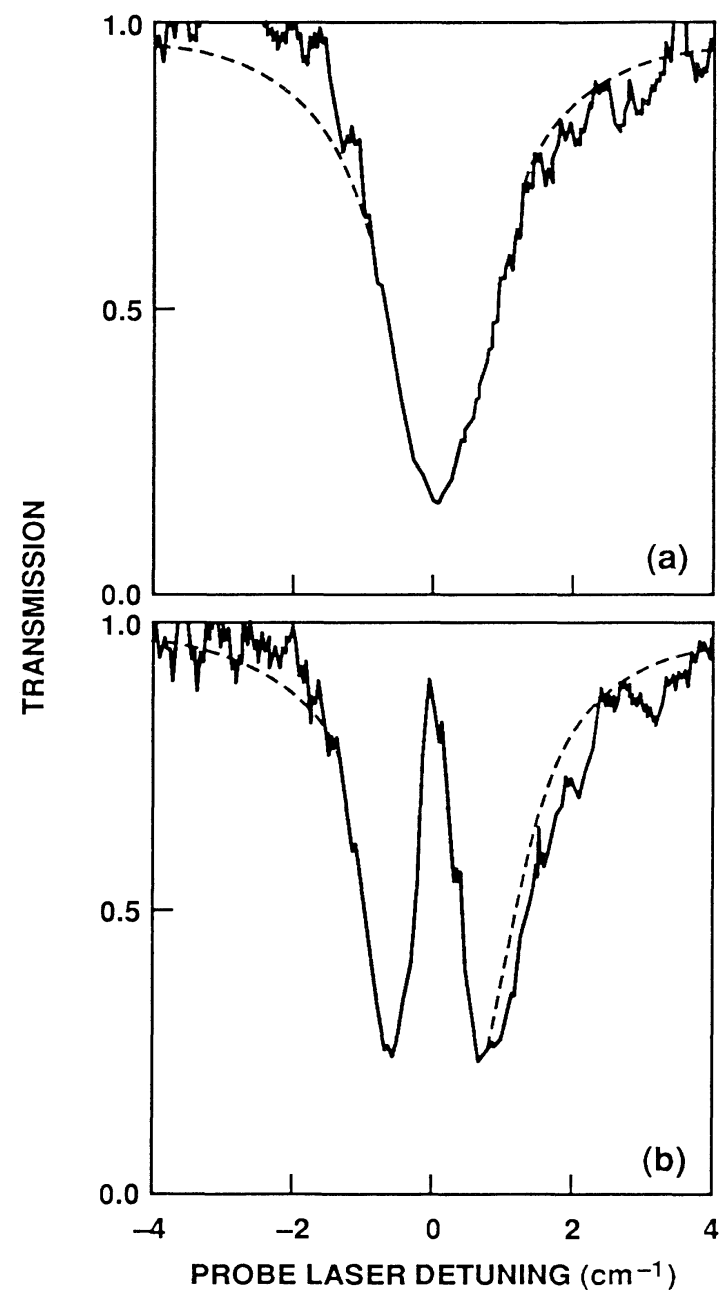

FIG. 2. Transmission vs probe laser detuning for (a) $\Omega_{23}=0$ and (b) $\Omega_{23}=1.3 \mathrm{~cm}^{-1}, \Delta \omega_{c}=-0.2 \mathrm{~cm}^{-1}$. Minimum transmission is $\exp (-1.7)$. 
$\mathrm{cm}^{-1}$ ), we determine an (autoionizing) linewidth of 1.2 $\mathrm{cm}^{-1}$, and a population-oscillator-strength-length product of $3.6 \times 10^{12} \mathrm{~cm}^{-2}$. Figure 2 (b) shows transmission versus probe frequency at a coupling-laser intensity which corresponds to a Rabi frequency on the $4 d 5 p^{1} D_{2}-4 d 5 d^{1} D_{2}$ transition of $1.3 \mathrm{~cm}^{-1}$. This Rabi frequency is estimated by measuring the frequency spacing of the two transmission minima. This spacing varies as the square root of the measured coupling-laser intensity and agrees with the calculated oscillator strength. We calculate $\Omega=0.42\left(P_{c} / A\right)^{1 / 2}$, for $\Omega$ in $\mathrm{cm}^{-1}, P_{c}$ in $\mathrm{MW}$, and $A$ in $\mathrm{cm}^{2}$.

Figure 3 shows transmission as a function of the probe frequency with the $5 s 5 p{ }^{1} P_{1}$ level population sufficiently large that the transition is optically thick. In Fig. 3(a), the coupling laser is off. By fitting with a Voigt profile and using the autoionizing linewidth determined from Fig. 2 we determine a minimum transmission of $\exp (-20 \pm 1)$. [We measure directly to $\exp (-7)$ ]. Figure 3(b) shows transmission with a coupling-laser Rabi frequency of $1.5 \mathrm{~cm}^{-1}$. We observe a change in transmitted intensity from $\exp (-20 \pm 1)$ without the coupling laser to $\exp (-1.0 \pm 0.1)$ with the coupling laser present.

In Figs. 2 and 3 the experimental results are compared with theory (dashed curve). For monochromatic laser fields, without Doppler broadening, the absorption cross section is 10,11

$$
\begin{aligned}
\frac{\sigma}{\sigma_{0}} & =\frac{\left(\Delta \omega_{p}-\Delta \omega_{c}\right)^{2} \gamma_{31}^{2}+\gamma_{21} \gamma_{31}\left(\Omega_{23}^{2} / 4+\gamma_{21} \gamma_{31}\right)}{\left[\Omega_{23}^{2} / 4+\gamma_{21} \gamma_{31}-\left(\Delta \omega_{p}-\Delta \omega_{c}\right) \Delta \omega_{p}\right]^{2}+\left[\Delta \omega_{p}\left(\gamma_{21}+\gamma_{31}\right)-\Delta \omega_{c} \gamma_{31}\right]^{2}}, \\
\sigma_{0} & =\frac{2}{\hbar}\left(\frac{\mu_{0}}{\epsilon_{0}}\right)^{1 / 2} \frac{\omega_{31}\left|\mu_{31}\right|^{2}}{\gamma_{31}} .
\end{aligned}
$$

Here, $\sigma_{0}$ is the peak absorption cross section of the $|1\rangle$ $|3\rangle$ transition in the absence of the coupling laser; $\Delta \omega_{p}$ $=\omega_{3}-\omega_{p}-\omega_{1}$ and $\Delta \omega_{c}=\omega_{3}-\omega_{c}-\omega_{2}$ are the detunings of the probe and coupling lasers; $\omega_{31}=\omega_{3}-\omega_{1}, \Omega_{23}$ is the Rabi frequency of the coupling laser, $\gamma_{31}=\Gamma_{3} / 2$ $+\Gamma_{1} / 2+\gamma_{31}$ deph, and $\gamma_{21}=\Gamma_{2} / 2+\Gamma_{1} / 2+\gamma_{21}$ deph. $\Gamma_{3}, \Gamma_{2}$, and $\Gamma_{1}$ are the decay rates of states $|3\rangle,|2\rangle$, and $|1\rangle$, respectively, and $\gamma_{31 \text { deph }}$ and $\gamma_{21 \text { deph }}$ are the macroscopic dephasing rates of the corresponding transitions.

It is because the peak absorption cross section $\sigma_{0}$ $=2 \times 10^{-14} \mathrm{~cm}^{-2}$ as compared to a background cross section of $2 \times 10^{-16} \mathrm{~cm}^{2}$ (measured within $\pm 40 \mathrm{~cm}^{-1}$ from line center, with no additional spectral structure) that we are able to use the simpler equation of Ref. 10 rather than the results of Refs. 1-3, which include the interference with the photoionization channel to the continuum. The parameters used in the theoretical curves are $\gamma_{31} \cong \Gamma_{3} / 2=0.6 \mathrm{~cm}^{-1}$, determined from Fig. 2(a), and $\gamma_{21}=0.025 \mathrm{~cm}^{-1}$ determined from Fig. 2(b). This latter parameter provides a reasonable fit to the experimental data, and is meant to include the contributions of collisional dephasing and coupling-laser linewidth. ${ }^{12}$ The theoretical (dashed) curves of Fig. 3 involve no further fit. With both the coupling and probe lasers circularly polarized, the overall $m$-state-degenerate level system of Fig. 1 may be viewed as three independent threestate systems. The matrix elements of the probe transition for the three systems have the ratio 0.58:1.0:1.42, while those for the coupling transition have the ratio 1.0:1.0:0.82. The theoretical curves in Figs. 2 and 3 sum over these subsystems with the assumption that all $m_{J}$ states of level $|1\rangle$ have equal population. These curves are also averaged to include the effects of the $0.03-\mathrm{cm}^{-1}$ Doppler width and the $0.15-\mathrm{cm}^{-1}$ probe-laser linewidth.

There is a consistent asymmetry in the experimental curves which is not accounted for by Eq. (1a). This is probably the result of an additional Fano interference with a broader and weaker autoionizing line. By assuming a single (totally interfering) photoionization channel with a cross section of $2 \times 10^{-16} \mathrm{~cm}^{2}$ we achieve a good fit to the experimental curves in the line wings. We note that an additional interference of this type does not limit the maximum transparency, but does cause a small shift in the frequency at which it occurs. For the coupling Rabi frequencies used, we estimate that this shift is at most $0.1 \mathrm{~cm}^{-1}$. There also may be additional noninterfering and therefore noncancelable photoionization channels.

The transparency in an experiment of this type results from a combination of the ac-Stark splitting of two dressed states and the destructive interference in the absorption to these states. For the data of Fig. 3(b), if the states did not interfere, but were still split by $1.5 \mathrm{~cm}^{-1}$, the maximum transmission would be $\exp (-7)$ instead of, as observed, $\exp (-1)$; i.e., if it were not for the interference the transmission window would be unobservable. For the destructive interference between the dressed states to be important the ac-Stark splitting must be on order of their autoionizing linewidth. It is for this reason that the present experiment was chosen.

We remark that a first attempt at this experiment failed. This failure was due to (what at that time was) a multimode coupling laser. Observation of an electromagnetically induced transparency requires a temporally smooth coupling laser.

The observation of electromagnetically induced transparency does not require coherence of the probe laser and may be observed with white light and a monochromator. Also, it is not required that bare state $|3\rangle$ decay 


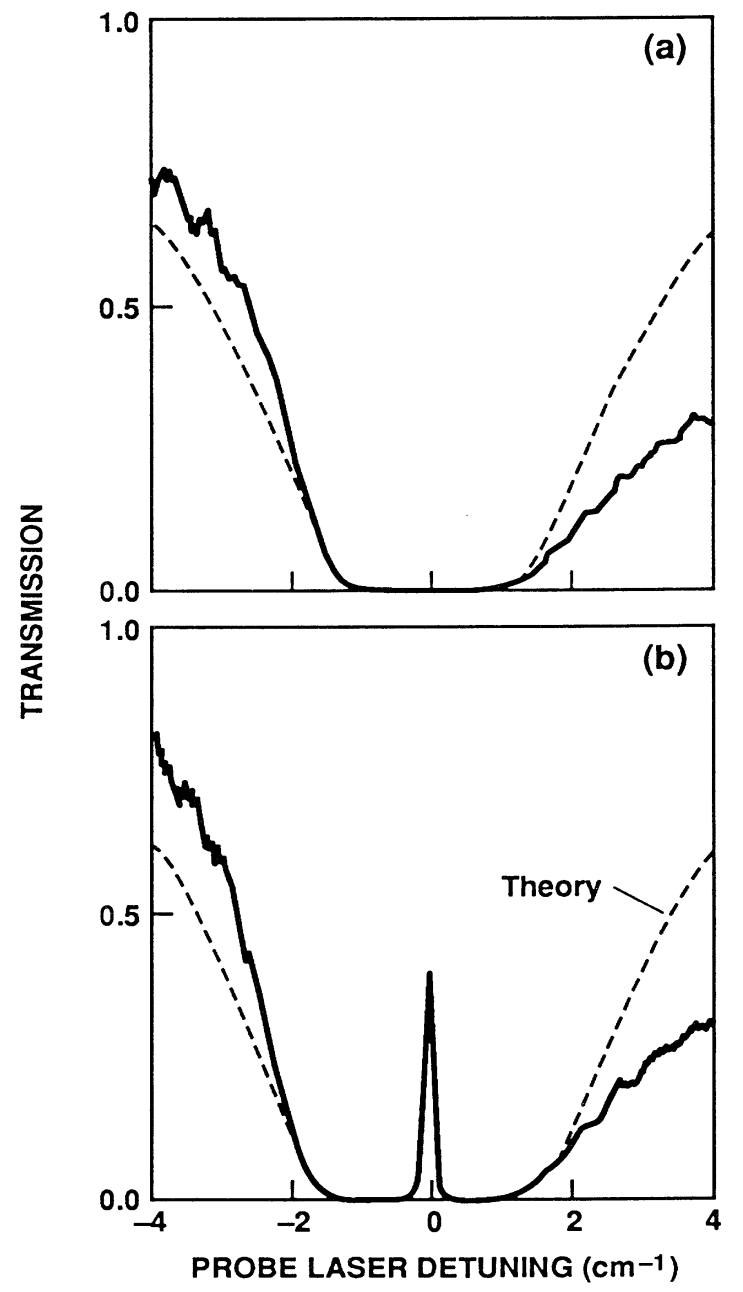

FIG. 3. Transmission vs probe laser detuning for (a) $\Omega_{23}=0$ and (b) $\Omega_{23}=1.5 \mathrm{~cm}^{-1}, \Delta \omega_{c}=-0.1 \mathrm{~cm}^{-1}$. Minimum transmission is $\exp (-20)$.

to a single channel; to the extent that state $|2\rangle$ is metastable, the dressed states have the same weight per channel decay and therefore the same interference profile.

In summary we have shown how an opaque optical medium may be rendered transparent by means of a destructive interference which is caused by applying an additional electromagnetic field. It has recently been predicted that this type of destructive interference is not present in the emissive profile of the species. This work is therefore an essential step in the implementation of inversion-free lasers, ${ }^{10}$ Raman devices, ${ }^{13}$ and multiply resonant nonlinear optical devices which make use of this transparency. ${ }^{11,14,15}$ This type of transparency is apt to be extendable to resonance-free photoionization continua ${ }^{4}$ and in some cases to collisionally broadened bound levels. ${ }^{10,11}$

We thank P. Corkum, A. Kasapi, J. E. Field, K. H. Hahn, and Guang-Yu Yin for helpful discussions. One of us (K.-J.B.) thanks the Deutsch Forschungsgemeinschaft for support. This work was jointly supported by the U.S. Air Force Office of Scientific Research, the U.S. Army Research Office, the U.S. Office of Naval Research, and the Strategic Defense Initiative Organization.

'P. Lambropoulos and P. Zoller, Phys. Rev. A 24, 379 (1981); H. Bachau and P. Lambropoulos, Phys. Rev. A 34, 4785 (1986).

${ }^{2}$ K. Rz̧ażewski and J. H. Eberly, Phys. Rev. Lett. 47, 408 (1981); Z. Deng and J. H. Eberly, J. Opt. Soc. Am. B 1, 102 (1984).

${ }^{3}$ S. Ravi and G. S. Agarwal, Phys. Rev. A 35, 3354 (1987); S. L. Haan and G. S. Agarwal, Phys. Rev. A 35, 4592 (1987).

${ }^{4}$ P. L. Knight, M. A. Lauder, and B. J. Dalton, Phys. Rep. 190, 1 (1990).

${ }^{5}$ Yu. I. Heller, V. F. Lukinykh, A. K. Popov, and V. V. Slabko, Phys. Lett. 82A, 4 (1981); L. I. Pavlov, S. S. Dimov, D. I. Metchkov, G. M. Mileva, and K. V. Stamenov, Phys. Lett. 89A, 441 (1982); M. H. R. Hutchinson and K. M. M. Ness, Phys. Rev. Lett. 60, 105 (1988); E. B. Saloman, J. W. Cooper, and D. E. Kelleher, Phys. Rev. Lett. 55, 193 (1985).

${ }^{6}$ R. M. Whitley and C. R. Stroud, Jr., Phys. Rev. A 14, 1498 (1976); G. Alzetta, A. Gozzini, L. Mopi, and G. Orriols, Nuovo Cimento B 36, 5 (1976).

${ }^{7}$ G. H. Newsom, S. O'Connor, and R. C. M. Learner, J. Phys. B 6, 2162 (1973).

${ }^{8}$ R. D. Cowan, The Theory of Atomic Structure and Spectra (Univ. California Press, Berkeley, 1981), Secs. 8-1, 16-1, and 18-7.

${ }^{9}$ M. G. Littman, Appl. Opt. 23, 4465 (1984).

${ }^{10}$ A. Imamoğlu and S. E. Harris, Opt. Lett. 14, 1344 (1989); S. E. Harris, Phys. Rev. Lett. 62, 1033 (1989).

${ }^{11}$ S. E. Harris, J. E. Field, and A. Imamoğlu, Phys. Rev. Lett. 64, 1107 (1990).

${ }^{12}$ G. S. Agarwal, Phys. Rev. A 18, 1490 (1978).

${ }^{13}$ G. S. Agarwal, S. Ravi, and J. Cooper, Phys. Rev. A 41, 4727 (1990).

${ }^{14}$ Surya P. Tewari and G. S. Agarwal, Phys. Rev. Lett. 56, 1811 (1986).

${ }^{15}$ K. Hakuta, L. Marmet, and B. P. Stoicheff, Phys. Rev. Lett. 66, 596 (1991). 